\title{
RECONOCIMIENTO DE FACHADAS MEDIANTE REALIDAD AUMENTADA COMO MEDIO PUBLICITARIO
}

\section{FACADE RECOGNITION BY AUGMENTED REALITY AS AN ADVERTISING WAY}

\author{
Álvaro Caiza Infante ${ }^{1}$
}

Dario Robayo Jácome ${ }^{2}$

Ricardo Patricio Medina Chicaiza ${ }^{3}$

1. Ingeniero de Sistemas y Computación. Escuela de Ingeniería en Sistemas. Pontificia Universidad Católica del Ecuador Sede Ambato. Ecuador. E-mail: alvaro htm@hotmail.com

2. Docente titular de la Escuela de Ingeniería en Sistemas. Pontificia Universidad Católica del Ecuador Sede Ambato. Ecuador. E-mail: drobayo@pucesa.edu.ec

3. Ingeniero de Sistemas y Computación. Magister en Tecnologías de la Información. Pontificia Universidad Católica del Ecuador, Docente de la Escuela de Ingeniería en Sistemas. Universidad Técnica de Ambato, Docente de la Facultad de Ciencias Administrativas, Ambato - Ecuador. Correo: pmedina@pucesa.edu.ec; ricardopmedina@uta.edu.ec 


\section{RESUMEN}

El presente artículo tiene como objetivo utilizar la realidad aumentada (RA) para la implementación de una aplicación móvil en sistema operativo Android como estrategia informativa publicitaria. La investigación evidencia como problema, la insuficiencia en la utilización de RA en la publicidad para su uso en dispositivos móviles. La revisión de fundamentos teóricos presenta conceptos de publicidad y varias confusiones que se tiene sobre RA y lo que ésta representa; además, un análisis comparativo que apoye al usuario a seleccionar la herramienta de desarrollo adecuada. Entre los resultados relevantes de la etapa de diagnóstico se tiene una alta expectativa para acceder a información en demanda a través de medios no tradicionales, como dispositivos móviles; esto lleva a la creación de nuevos espacios publicitarios que cubran dichas expectativas. Los resultados obtenidos junto a la revisión de antecedentes en el área temática, propone como producto final una aplicación informativa publicitaria bajo RA para la Pontificia Universidad Católica del Ecuador Sede Ambato (PUCESA), a través de la cual los usuarios podrán acceder de distintas maneras, a información relevante de la institución.

\section{ABSTRACT}

This article aims to use the augmented reality (AR) for the implementation of a mobile application on Android operating system as an advertising information strategy. The research evidence as a problem, the failure in the use of AR in advertising the use of it on mobile devices. The revision of the theoretical fundaments presents several advertising concepts and confusions on AR use and what represents; in addition, a comparative analysis that supports a user to select an adequate developed tool. Among the relevant results of the diagnostic stage a high expectation is being looked forward to accessing information on demand through non-traditional media such as mobile devices; this leads to the creation of new advertising spaces that fill these expectations. The results obtained with the background revision in the proposed theme, as final product an advertising informative application is proposed under AR for the Pontificia Universidad Catolica del Ecuador Ambato (PUCESA), through which users can access by different ways to relevant information of the institution.

\section{PALABRAS CLAVE}

Realidad Aumentada; Publicidad; Mercadeo; Dispositivo Móvil.

\section{KEY WORDS}

Augmented Reality; Advertising; Marketing; Mobile Device. 


\section{INTRODUCCIÓN}

Las aplicaciones RA particularmente para dispositivos móviles no son muy comunes en la actualidad, de acuerdo a X. Basogain, M. Olabe, K. Espinosa, C. Rouèche, \& J.C. Olabe, (2008) está relacionada con la Realidad Virtual (RV), área que es más conocida; la principal diferencia es que la RA no reemplaza el mundo real por uno virtual, sino al contrario, mantiene el mundo real que ve el usuario complementándolo con información virtual superpuesta al real.

El uso de teléfonos inteligentes (smartphones) se ha convertido en parte inevitable e inconsciente en la sociedad moderna, navegar es una tarea que se repite varias veces en el día y muy común en los consumidores, factor al que las redes sociales han impulsado notoriamente. Por ello la publicidad en su evolución, ha llegado a ser parte de los contenidos en los teléfonos móviles, los investigadores Román, González-Mesones, \& Marinas, (2005), el principal público objetivo de este medio son los adolescentes, jóvenes y adultos de hasta 35 años (intervalo que irá aumentando con el paso del tiempo), siendo así difícil de alcanzarlos con publicidad por otros medios. Estos nuevos servicios podrán mejorar la RA aumentando la atracción de los usuarios y abriendo nuevas estrategias de publicidad, brindando una experiencia de usuario avanzada en RA móvil (Prado E., 2010).

En el caso del Ecuador la RA es un tema poco conocido tanto en el uso como en el desarrollo de aplicaciones móviles que utilicen este tipo de tecnología; en el tema del marketing y publicidad existen pocos trabajos documentados como por ejemplo la agencia Geeks Ecuador que se inició desarrollando prototipos de tarjetas de presentación y en el mes de marzo de 2011 realizaron una campaña de marketing digital para el Mall del Sol de la ciudad de Guayaquil, denominada "Días de Dinosaurios" misma que incluía la impresión de gafas especiales y a través de RA podían proyectar el rostro de un dinosaurio (Chimbo \& Tierra, 2012). De forma parecida agencias como Camaleón Diseño Visual y Blackbox Ecuador, ofrecen en sus portales web servicios de publicidad mediante RA.

Por lo expuesto anteriormente, surge la necesidad de revisar herramientas de desarrollo de este tipo de aplicaciones, las cuales se utilizarán en la creación de un medio publicitario para la PUCESA, que permita una nueva forma de llegar a este nicho de mercado facilitando el acceso a la información a través de dispositivos modernos. 


\section{FUNDAMENTOS TEÓRICOS}

\section{REALIDAD AUMENTADA}

La sociedad actual está experimentando cambios que favorecen la aparición de nuevas formas de comunicación, entre ellas, los dispositivos móviles, que permiten maneras diferentes de llegar con información a varios grupos de personas. Una de las tecnologías que aún no son aprovechadas por estos dispositivos, es precisamente la RA, que solamente está siendo explotada a nivel de computadores. A criterio de los autores en el medio aún no tiene una idea clara de lo que en realidad es la RA, e incluso se tiende a confundir con los conceptos de realidad virtual. De acuerdo a Mullen, (2011), el término realidad aumentada se utiliza para describir una combinación de tecnologías que permiten la mezcla en tiempo real de contenido generado por computadora con tomas de video en vivo. Tradicionalmente, se distingue de la RV en que esta implica la creación de entornos 3D completos e inmersión, y la RA utiliza diversas tecnologías de hardware para crear una versión aumentada o compuesta basada en el mundo real.

Según Madden, (2011), la RV sumerge al usuario en un mundo generado por computadora mientras que la RA combina el mundo real con gráficos por computador. A diferencia de la $\mathrm{RV}$, que requiere equipo especializado para ser experimentado, RA requiere solamente una manera de capturar el mundo que le rodea y los medios para experimentar el mundo de la informática (por lo general mediante la superposición de gráficos por computador en la imagen capturada por la cámara).

Para Arroyo, (2011), a diferencia de la RV, que supone un mayor componente de virtualidad que de realidad, la RA implica la superposición de objetos (imagen, vídeo o sonido) y datos digitales sobre la realidad. Por lo tanto, podríamos decir que se trata de una forma de visualización enriquecida del entorno que nos rodea.

De Pedro Carracedo \& Méndez, (2012), mencionan que un aspecto importante de la RA lo constituye la interfaz con el usuario, ya que en la mayoría de las aplicaciones las capas superpuestas sobre la imagen real corresponden a vídeo y sonido, lo que en cualquier caso, proporciona un mundo completamente "inmersivo", característico de la RV, pero también un mundo físico, en el que los usuarios disponen de cierta capacidad para interaccionar con el medio. Finalmente, la Fundación Telefónica de España menciona: "Bajo el paraguas de la RA se agrupan aquellas tecnologías que permiten la superposición, en tiempo real, de imágenes, marcadores o información generados virtualmente, sobre imágenes del mundo real" (Telefónica, 2011).

En base a lo descrito, queda claro que la RV hace uso de entornos 3D totalmente inmersivos para el usuario, con una nula posibilidad de interacción; mientras que la RA solamente hace uso de una superposición de información virtual sobre imágenes del mundo real, principalmente vídeo. Además, en cuanto a los requerimientos de hardware la diferencia es sustancial, ya que los recursos necesarios para software RV son muy ambiciosos, en tanto que para RA estos son mínimos; debido a esto, muchos de los teléfonos inteligentes de hoy en día se constituyen en dispositivos ideales para RA. 
Se vive en un mundo inundado de publicidad, según Medina \& Buil, (2013), existen varias campañas de marketing que pueden ser aplicadas a una empresa, sin embargo, se destaca la importancia de la publicidad en dispositivos móviles y el Internet, se menciona que aquí está el futuro ya que se puede llegar a consumidores en todo el mundo y con las plataformas existentes esta tarea se ha facilitado. Telefónica, (2011), analiza a la realidad aumentada en el ámbito publicitario, se llega a la conclusión que una campaña con esta tecnología dejará en la mente del cliente un recuerdo de la marca, ya que genera un gran impacto al permitirle interactuar directamente con el producto o servicio que ofrece la empresa. A criterio de los autores, una campaña publicitaria con realidad aumentada significa una ventaja competitiva enorme, pues inmiscuir al cliente en plataformas y tecnologías actuales genera nuevas y mejores experiencias en su cerebro con la firma de la marca o empresa.

Aumentar el valor del negocio y atraer a nuevos clientes son metas fijas en cualquier empresa, la RA es usada por las empresas para dar publicidad en línea a sus nuevos productos (Telefónica, 2011). Esta nueva forma de publicidad, ha sido adoptada por ejemplo, en el campo automovilístico, pues en Sood (2012) se muestran ejemplos de grandes empresas como BMW, Nissan, Toyota y Mini, que han usado las ventajas de esta tecnología para dar a conocer sus nuevos prototipos o jugar con la imaginación del usuario brindándole la experiencia de visualizar un modelo en 3D sobre una revista con opciones interactivas.

En el campo de la moda y el estilo las empresas no se quedan atrás a la hora de aprovechar todo lo que ofrece la realidad aumentada. Telefónica,(2011), reconoce su uso como algo innovador en esta área donde las personas ya no necesitan "probarse" físicamente la ropa antes de comprarla, pues gracias a esta tecnología es posible que el cliente visualice como le quedaría una o varias prendas con el único requerimiento de que se muestre ante una cámara. Esta forma de publicidad ahorra tiempo a los clientes a la vez que brinda entretenimiento al momento de experimentar con RA.

De lo anterior se evidencia que, para llegar a un potencial público objetivo a través de las técnicas conocidas de marketing, estas deben evolucionar en nuevas estrategias apoyadas en medios tecnológicos modernos, lo que por una parte despierta un mayor interés en los consumidores, por otro lado, al no permitir que la información llegue sin el consentimiento del usuario, facilita que el acceso a la misma sea en demanda bajo sus propias necesidades.

Al ser los jóvenes los principales usuarios de teléfonos inteligentes (Prado E., 2010), este sector es el que más interesa a las marcas ya que sus dispositivos, ayudan a implementar nuevas estrategias de marketing en línea, con aplicaciones como las derivadas de RA, lo que junto al acceso a redes sociales, ofrecen una gama de opciones, permitiendo también la identificación de nuevas necesidades de los usuarios. 


\section{RESULTADOS}

En el desarrollo de la metodología Mobil-D, se cumplió con las actividades propuestas en cada fase, las cuales se detalla a manera general:

\section{FASE DE EXPLORACIÓN}

Existen 2 partes interesadas o usuarios en este proyecto:

Dirección de Estudiantes de la PUCESA: Al ser el área que se encarga de la publicidad de la Universidad, y los requerimientos específicos fueron señalados en este departamento.

Estudiantes de último año de colegio: Por estar a punto de empezar una carrera universitaria pueden optar por la PUCESA para continuar con sus estudios.

En el análisis de los requerimientos, se obtuvo las siguientes características:

La aplicación será desarrollada para dispositivos Android. En la pantalla inicial se mostrará las instrucciones para usar la aplicación, y en la misma debe existir un botón para iniciar la cámara del dispositivo. La aplicación usará la cámara del dispositivo móvil, la misma deberá escanear objetos para su funcionamiento. Se deberá escanear la fachada de los distintos edificios dentro de la Universidad y se mostrarán en tiempo real modelos en 3D representativos a cada escuela o departamento que trabaje dentro de cada edificio. En cada escena de realidad aumentada existirán botones con acceso a escenas en 2D con información ampliada de la escuela o departamento seleccionado. Se podrá utilizar la aplicación durante el día en las instalaciones de la PUCESA para poder desplegar la información y objetos en 3D. La aplicación será descargable desde la tienda en línea Google Play o un link externo.

Por otra parte, se realizó el análisis de algunas herramientas de autor, en base a varios criterios de Sanz (2014) y Moralejo (2014). Varnum (2014) menciona sobre las particularidades que debe tener una herramienta de RA para que sea útil y se proyecte a futuro. Para Sood (2012) es importante el número de usuarios a la hora de realizar una aplicación, pues al ser una tecnología que se ha tomado su tiempo en darse a conocer, no existen muchas herramientas ni desarrolladores. Mullen (2011) y Furth (2011) proponen sobre los distintos entornos disponibles para hacer prototipos de realidad aumentada, sus tipos de licencia, los códigos útiles para programar este tipo de aplicaciones y coinciden en que la documentación y el soporte son un factores clave a la hora de seleccionar una herramienta. Los criterios definidos son:

- Tipo de licencia. - Herramienta libre o pagada.

- Entorno de Desarrollo Integrado (IDE de programación). - La compatibilidad del software development kit con aplicaciones que permitan compilar el archivo .apk.

- Multiplataforma. - La aplicación puede ser creada para la web u otras plataformas como iOS o Windows Phone. 
- Código de programación. - No todos los SDK permiten el desarrollo en cualquier tipo de código.

- Documentación. - La información proporcionada por cada herramienta, así como el soporte técnico brindado.

- Número de usuarios. - Cantidad de usuarios que utilizan o han utilizado la herramienta.

Se seleccionaron tres herramientas de desarrollo para RA, aquellas que gozan de una mayor popularidad: Vuforia, ARToolKit y Layar. Se dio una puntuación del 1 al 3 en los parámetros, para finalmente obtener un resultado total de cada uno y determinar la mejor opción. La calificación se basó en el grado de utilidad de cada parámetro para el proyecto, así: 1 Poco útil, 2 Medianamente útil, 3 Muy útil.

Las características de las aplicaciones, de acuerdo a los parámetros determinados se muestran a continuación:

\begin{tabular}{|c|c|c|c|}
\hline Parámetros/SDK & ARToolKit & Vuforia & Layar \\
\hline Tipo de Licencia & Gratuita & Gratuita y pagada & Pagada \\
\hline $\begin{array}{l}\text { Entorno de } \\
\text { Desarrollo } \\
\text { Integrado (IDE de } \\
\text { programación) }\end{array}$ & $\begin{array}{l}\text { Propio, integrable } \\
\text { con Android Studio }\end{array}$ & $\begin{array}{l}\text { Integrable con } \\
\text { Unity, Eclipse y } \\
\text { XCode }\end{array}$ & $\begin{array}{l}\text { Propio, integrable } \\
\text { con Android Studio, } \\
\text { Eclipse y PhoneGap }\end{array}$ \\
\hline Multiplataforma & $\begin{array}{c}\text { Desarrollo para: } \\
\text { Web } \\
\text { Android }\end{array}$ & $\begin{array}{c}\text { Desarrollo para: } \\
\text { Android } \\
\text { IOS } \\
\text { Web }\end{array}$ & $\begin{array}{c}\text { Desarrollo para: } \\
\text { Android } \\
\text { IOS } \\
\text { Web }\end{array}$ \\
\hline $\begin{array}{l}\text { Código de } \\
\text { Programación }\end{array}$ & C, Java, Matlab & Java, C\#, C++ & $\begin{array}{l}\text { Html (PhoneGap), } \\
\text { Java }\end{array}$ \\
\hline Documentación & $\begin{array}{c}\text { ARToolKit } \\
\text { Documentation }\end{array}$ & $\begin{array}{c}\text { Vuforia Developer } \\
\text { Library }\end{array}$ & $\begin{array}{l}\text { Layar Developer } \\
\text { Documentation, } \\
\text { Layar Help Center }\end{array}$ \\
\hline Número de usuarios & +3000 & +175000 & +100000 \\
\hline
\end{tabular}

Los resultados del análisis comparativo se resumen en la siguiente tabla:

\begin{tabular}{|l|c|c|c|}
\hline \multicolumn{1}{|c|}{ Parámetros/SDK } & ARToolKit & Vuforia & Layar \\
\hline Tipo de Licencia & 3 & 3 & 2 \\
\hline $\begin{array}{l}\text { Entorno de Desarrollo } \\
\text { Integrado (IDE de } \\
\text { programación) }\end{array}$ & 3 & 3 & 3 \\
\hline Multiplataforma & 2 & 3 & 3 \\
\hline Código de Programación & 3 & 3 & 2 \\
\hline Documentación & 2 & 3 & 3 \\
\hline Número de usuarios & 1 & 3 & 16 \\
\hline Sumatoria total & 14 & 18 & \\
\hline
\end{tabular}

Fuente: Elaboración propia 
Una vez determinado que Vuforia es el SDK para el desarrollo, seleccionó Unity como la herramienta de programación. Al estar la aplicación dirigida dispositivos Android se elimina la opción de XCode y al ser una aplicación de publicidad también es la adecuada, ya que permite manipular objetos en 3D de una manera más fácil e intuitiva que con Eclipse. Además, se puede exportar el proyecto a varias plataformas con una licencia gratuita que se usa para el desarrollo de aplicaciones sin fines de lucro. Todos los conocimientos necesarios para aprender a usar esta herramienta se encuentran en el sitio web Unity Documentation.

\section{FASE DE INICIALIZACIÓN}

Se realizó una revisión y refinamiento de los requerimientos y así tenerlos como base para la planificación del desarrollo. Se considera la línea de arquitectura en: desarrollar escena de inicio, desarrollar escenas de realidad aumentada, desarrollar escenas de información; las mismas que son cumplidas en su totalidad.

\section{FASE DE DESARROLLO}

Se implementaron las funcionalidades requeridas en el producto programando en ciclos de desarrollo iterativos e incrementales. Esta fase se dividió en entregas, en cada una de las cuales se realizaron las pruebas de aceptación. Se cumplió con sus partes, siendo: día de planificación, día de trabajo y día de entrega. Con las aplicaciones, se diseñó y construyó la aplicación RA móvil para la PUCESA. Esta fue desarrollada para que el usuario acceda a la información de la Universidad, activando mediante un escaneo del logotipo (Gráf. 1) o de las fachadas de los diferentes edificios del campus (Gráf. 2).

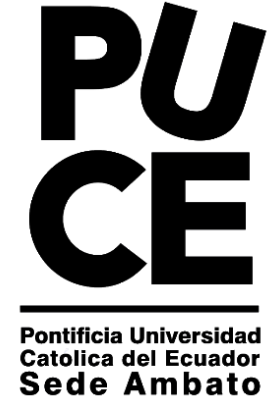

Gráfico 1. Logotipo PUCESA

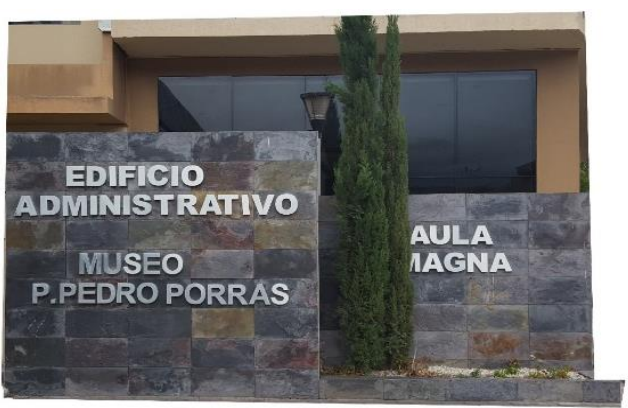

Gráfico 2. Edificio Administrativo

Cuenta también con áreas y botones de acción (Gráf. 3) para la selección y acceso a la información requerida de cada una de las carreras que conforman la oferta académica, así como también datos sobre admisión, servicios, clubes, entre otros. 


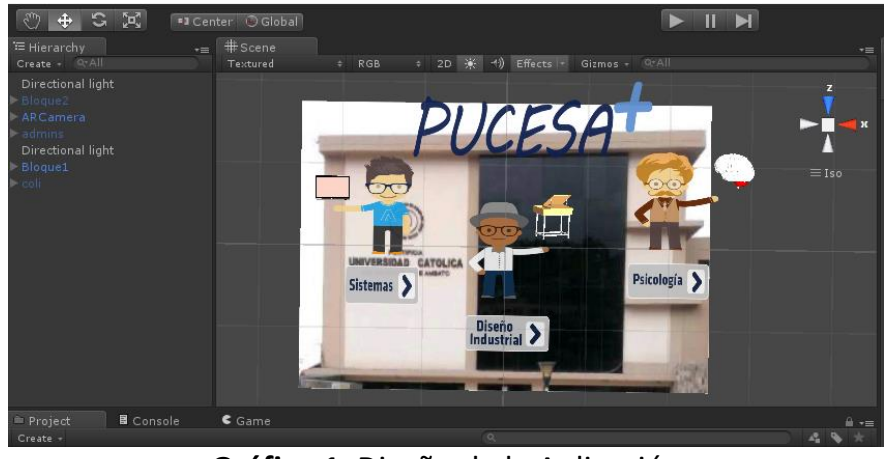

Gráfico 1. Diseño de la Aplicación

\section{FASE DE ESTABILIZACIÓN}

Se unieron los módulos o subsistemas y se obtuvo un solo producto final. Se considera la lista de deficiencias del primer entregable realizado en la fase de producción, la estabilización de la aplicación cuenta con los mismos pasos que la de producción, realizando un día de planificación, día de desarrollo y día de entrega, siguiendo los pasos en (Agile, 2006), además, se llena el primer taller post iteración, donde se considera: problema, acción, responsable, seguimiento del plan y realización.

Una vez concluida, revisada y aprobada por Dirección de Estudiantes, se publicó en la tienda Google Play Store (Fig. 4) para que pueda ser descargada e inmediatamente utilizada en cualquier dispositivo móvil con sistema operativo Android.

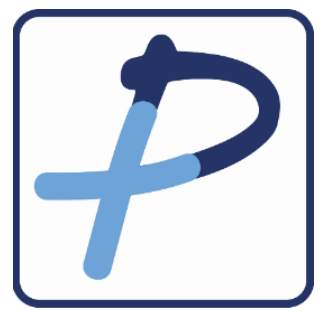

Gráfico 2. Logotipo de la aplicación en Google Play

\section{FASE DE PRUEBAS Y CORRECCIONES DEL SISTEMA}

Se realizó una búsqueda de errores latentes en el software con lo que se elaboró una nueva planificación, trabajo y entrega del software completo y funcional, que cumple con todos los requerimientos definidos en la primera fase. Además, se llena el segundo taller post iteración, donde se considera: problema, acción, responsable, seguimiento del plan y realización. 


\section{CONCLUSIONES}

En base al análisis comparativo se ha escogido al SDK de Vuforia por ser superior a las otras herramientas en aspectos como documentación, costo de licencia, entre otros; a través de la cual se ha podido desarrollar una aplicación móvil de RA para la Pontificia Universidad Católica del Ecuador Sede Ambato siguiendo la metodología ágil Mobile - D.

Las aplicaciones RA, particularmente para dispositivos móviles, están orientadas principalmente hacia un público objetivo joven, lo que hace que se pueda llegar a un número mayor de potenciales clientes para una determinada publicidad. Esto constituye una nueva estrategia de marketing que aprovecha las nuevas tecnologías para transmitir información importante.

La aplicación tiene una buena aceptación en la tienda Google Play Store lo cual se refleja en la cantidad de descargas y comentarios positivos recibidos. 


\section{REFERENCIAS}

Arroyo, N. (2011). Información en el móvil. Recuperado a partir de http://www.digitaliapublishing.com/a/20204/informaci-n-en-el-m-vil

Chimbo, E., \& Tierra, D. (2012). Estudio de la Realidad Aumentada Aplicada al Diseño Arquitectónico de Modelados 3D en la Web ESPOCH. Recuperado a partir de http://dspace.espoch.edu.ec/handle/123456789/2061

Daniel, F., Papadopoulos, G. A., \& Thiran, P. (2013). Mobile Web Information Systems: 10th International Conference, MobiWIS 2013, Paphos, Cyprus, August 26-29, 2013, Proceedings. Springer.

De Pedro Carracedo, J., \& Méndez, C. L. M. (2012). Realidad Aumentada: Una Alternativa Metodológica en la Educación Primaria Nicaragüense. IEEE-RITA, 7(2), 102-108.

Furth, B. (2011). Handbook of Augmented Reality. Springer Science \& Business Media.

Madden, L. (2011). Professional Augmented Reality Browsers for Smartphones: Programming for junaio, Layar and Wikitude. Hoboken, NJ, USA: Wiley. Recuperado a partir de http://site.ebrary.com/lib/puce/docDetail.action?docID=10484850

Medina, P., \& Buil, P. (2013). La publicidad si vende. Gazol.

Moralejo, L. (2014). Análisis comparativo de herramientas de autor para la creación de actividades de realidad aumentada. Facultad de Informática. Recuperado a partir de http://sedici.unlp.edu.ar/handle/10915/43605

Mullen, T. (2011). Prototyping Augmented Reality. Hoboken, NJ, USA: Sybex. Recuperado a partir de http://site.ebrary.com/lib/puce/docDetail.action?doclD=10501260

Prado E. (2010). Telos 84: Contenidos digitales para la nueva televisión. Fundación Telefónica.

Román, F., González-Mesones, F., \& Marinas, I. (2005). Mobile MK: la revolución multimedia. ESIC Editorial.

Sanz, C. (2014). Material de estudio del curso "Tecnología Informática. Evolución y Aplicaciones» de la carrera de Especialización en Tecnología Informática Aplicada en Educación.

Sood, R. (2012). Pro Android Augmented Reality. Apress.

Telefónica, F. (2011). Realidad Aumentada: una nueva lente para ver el mundo. Fundación Telefónica.

Varnum, K. J. (2014). The Top Technologies Every Librarian Needs to Know: A LITA Guide. American Library Association.

X. Basogain, M. Olabe, K. Espinosa, C. Rouèche, \& J.C. Olabe. (2008). Realidad Aumentada en la Educación: una tecnología emergente. 